ORIGINAL PAPER

\title{
ARE THERE CHANGES IN ORGANOPHOSPHORUS POISONING TRENDS OVER A 10 YEARS PERIOD?
}

\author{
Monica Dumitrașcu ${ }^{1}$, Florentina Muşat ${ }^{2}$, R. A. Macovei ${ }^{1,2}$ \\ ${ }^{1}$ Clinical Emergency Hospital, Bucharest, Romania \\ ${ }^{2}$ The University of Medicine and Pharmacy "Carol Davila", Bucharest, Romania \\ Corresponding author: Monica Dumitrașcu \\ Email: sandalberto@gmail.com
}

\begin{abstract}
Organophosphates poisoning represents a challenge for the emergency medical specialist, the first who comes into contact with intoxicated patients and who should promptly manage this pathology. Our study aims to determine whether there are any significant changes after a 10 years period regarding some characteristics of the patients with acute organophosphorus compounds poisoning. The study was retrospective, observational, descriptive, non-randomized and took place at the Bucharest Emergency Clinical Hospital during 1st of January 1995 - 31st of December 1997 and 1st of January 2014 - 31st of December 2016 respectively. Our study was based on the comparative assessment of two groups of patients as follows: patients admitted during 1995-1997 (292 patients) and patients admitted during 2014-2016 (161 patients). The percentage of suicidal attempts through organophosphorus compounds decreased over the course of 10 years from $84.78 \%$ in 1996 to $63.63 \%$ in 2016. A psychiatric pathology was identified in $39.31 \%$ of the suicidal attempts and in $15.68 \%$ of accidental contact cases. Regarding the distribution of exposure type per year, we observed an increase in cases where the penetration pathway occurred through skin and inhalation from $9.57 \%$ in 1995 to $18.33 \%$ in 2015, with a proportional decrease in the digestive exposure. In the analyzed groups, 36 deaths were identified, with an equal distribution in the two studied periods, of about 8-10\%. Although there was a 10-year interval between the two patient lots during which intensive care techniques have significantly progressed, we observed from the statistical data that the death rate did not decrease significantly in the 2014-2016 lot compared to the 1994-1996 lot. We believe that this is partly due to the gravity of these patients and to the time elapsed from the presentation of the patients to the physician and appropriate treatment initiation.
\end{abstract}

KEYWORDS: organophosphorus compounds, poisoning, acetylcholinesterase

\section{INTRODUCTION}

Organophosphates (OP) poisoning represents a challenge for the emergency medical specialist, the first who comes into contact with intoxicated patients and who should promptly manage this pathology. Usually, it is easy to make the diagnosis, using anamnestic data both from the patients or their caregivers and recognizing the characteristic symptomatology. The degree of impairment is quantified based on the pseudocholinesterase 
activity measurements, the decrease of which is a reliable prognostic indicator for these patients.

Our study aims to determine whether there are any significant changes after a 10 years period regarding some characteristics of the patients with acute OP compounds poisoning.

\section{MATERIALS AND METHOD}

The study was retrospective, observational, descriptive, non-randomized and took place at the Bucharest Emergency Clinical Hospital during 1st of January 1995 - 31st of December 1997 and 1st of January 2014 - 31st of December 2016 respectively.

All the legal requirements and legal precautions in the European Union have been respected in terms of confidentiality, ethics and professional ethics.

Our study was based on the comparative assessment of two groups of patients as follows: - $\quad$ Patients admitted during 1995-1997 (292 patients)

- $\quad$ Patients admitted during 2014-2016 (161 patients)

\section{RESULTS}

We analyzed a total number of 453 patients admitted for acute poisoning with OP compounds during 1995-1997 and 2014-2016.

Distribution by years of the cases included in the study showed a moderate decrease in the number of patients admitted with a diagnosis of poisoning with OP. Thus, during 1995-1997 there were 292 cases, with a peak of 106 in 1997, while during 2014-2016, 161 patients were identified, with a maximum of 68 cases in 2014 (Figure 1).

Statistical data on the age of patients included in the study reveals an average of 42.40 years with a minimum of 15 years and a maximum of 89 years. The distribution is uneven with a median of 42 years and a confidence interval of 39-45 years (Table 1).

Distribution of the patients enrolled in the study, depending on the level of schooling, highlighted 57 cases with higher education (representing 12.6\%), 173 patients who graduated high school (38.2\%) and 223 cases having completed no more than 8 grades (representing 49.2\%).

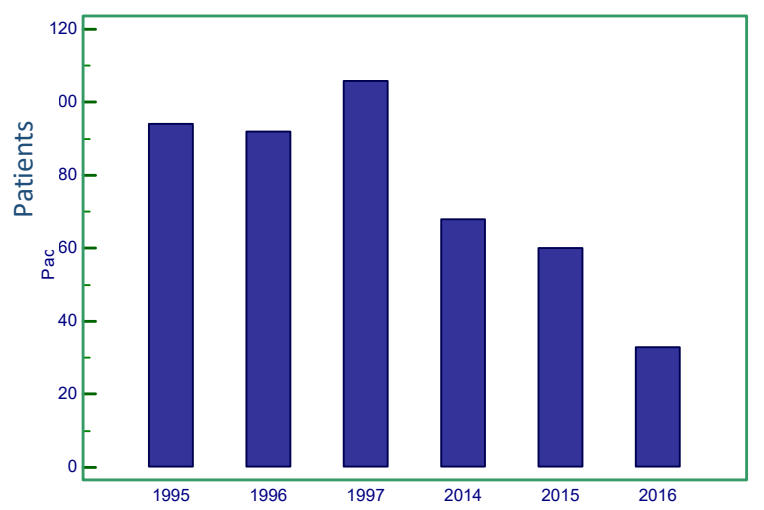

Figure 1 - Cases distribution according to year of admission

\begin{tabular}{ll}
\hline Sample size & 453 \\
\hline Lowest value & 15 \\
\hline Highest value & 89 \\
\hline Arithmetic mean & 42.40 \\
\hline 95\% CI for the mean & 40.82 to 43.97 \\
\hline Median & 42 \\
\hline 95\% CI for the median & 39 to 45 \\
\hline Standard deviation & 17.0539 \\
\hline Table 1 - Statistical data on patient age
\end{tabular}

Figure 2 points to an increase in the level of schooling from $7,44 \%$ of patients with higher education in 1995 to $21,21 \%$ in 2016 . The percentage of patients with maximum 8 grades was $53,19 \%$ in 1995 , to reach $36.36 \%$ in 2016.

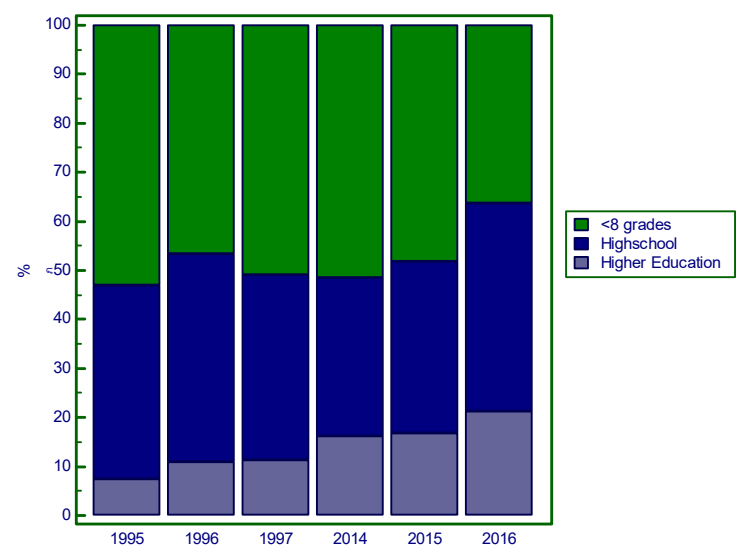

Figure 2 - Percentage distribution of cases according to patient education level and year of admission 
The patients have come into contact with OP compounds in two ways: accidentally and following a suicidal attempt. Of the total of 453 patients enrolled in the study, 102 cases $(22.5 \%)$ were accidental poisonings, while in 351 cases (77.5\%) poisoning was intentional (Table 2). The percentage of suicidal attempts decreased over the course of 10 years from $84.78 \%$ in 1996 to $63.63 \%$ in 2016 (Figure 3).

\begin{tabular}{lll}
\hline Accidental poisoning & 102 & $22.5 \%$ \\
\hline Suicidal attempt & 351 & $77.5 \%$ \\
\hline Total & 453 & $100.0 \%$ \\
\hline
\end{tabular}

Table 2 - cases distribution according to the contact type

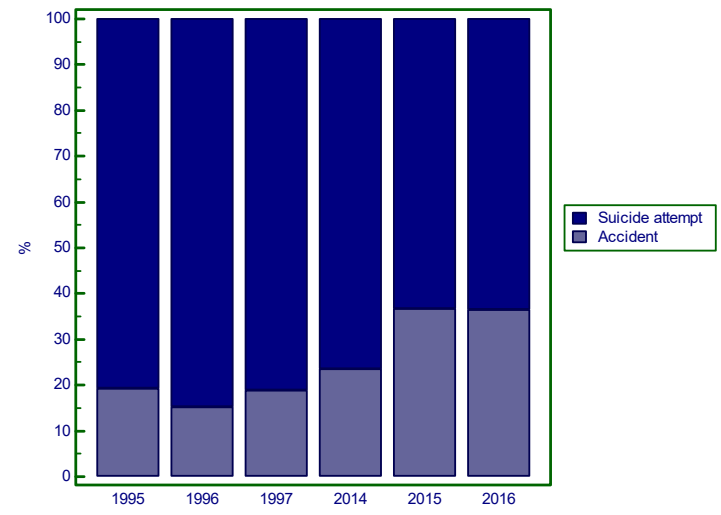

Figure 3 - Percentage distribution of cases according to the contact type and year of admission

A psychiatric pathology or a psychological disorder (Table 3) was not identified by anamnesis or clinically in 299 patients (representing 66\%). This percentage remained approximately the same in the two analyzed groups (Table 4).

\begin{tabular}{lll}
\hline No & 299 & $66.0 \%$ \\
\hline Yes & 154 & $34.0 \%$ \\
\hline Total & 453 & $100.0 \%$
\end{tabular}

Table 3 - Cases distribution according to the presence or absence of a psychiatric pathology

\begin{tabular}{lllllll}
\hline & 1995 & 1996 & 1997 & 2014 & 2015 & 2016 \\
\hline No & 65 & 57 & 70 & 44 & 42 & 21 \\
\hline Yes & 29 & 35 & 36 & 24 & 18 & 12 \\
\hline
\end{tabular}

Table 4 - Percentage distribution of cases according to the presence (Yes) or absence (No) of a psychiatric pathology and the year of admission
A psychiatric pathology was identified in $39.31 \%$ of the suicidal attempts and in $15.68 \%$ of accidental contact cases (Table 5).

\begin{tabular}{ccc}
\hline & \multicolumn{2}{c}{ Intoxication type } \\
\hline Psychiatric pathology & Accidental & $\begin{array}{c}\text { Suicidal } \\
\text { attempt }\end{array}$ \\
\hline No & 86 & 213 \\
\hline Yes & 16 & 138 \\
\hline
\end{tabular}

Table 5 - Percentage distribution of cases according to the presence or absence of a psychiatric pathology and the type of intoxication

OP compounds exposure occurred through dermal contact in 19 cases representing $4.2 \%$, inhalation in 30 cases representing $6.6 \%$ and ingestion in a majority of 404 cases representing $89.2 \%$ (Figure 4 ).

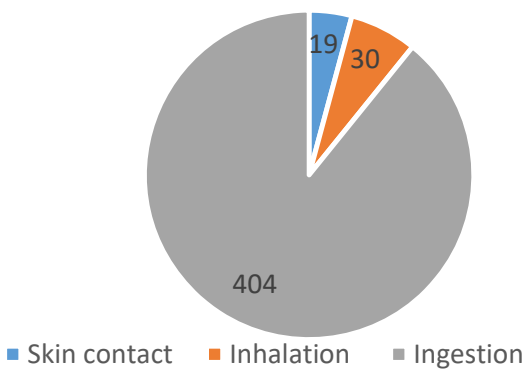

Figure 4 - Cases distribution according to exposure type

Regarding the distribution of exposure type per year, we observed an increase in cases where the penetration pathway occurred through skin and inhalation from $9.57 \%$ in 1995 to $18.33 \%$ in 2015 , with a proportional decrease in the digestive exposure (Figure 5).

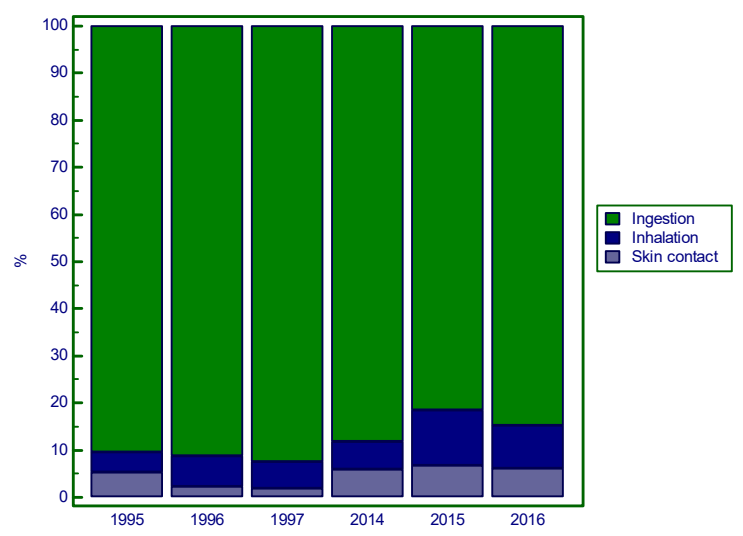

Figure 5- Percentage distribution of cases according to exposure type and year of admission 
The distribution of cases depending on the exposure type in relation to the cause of intoxication with OP compounds (Figure 6), highlights the fact that most of the digestive poisoning occurred as a result of a suicidal attempt $(84.40 \%)$, while the majority of the inhalation and skin contact exposures were accidental (83.33\% and $73.68 \%$ respectively).

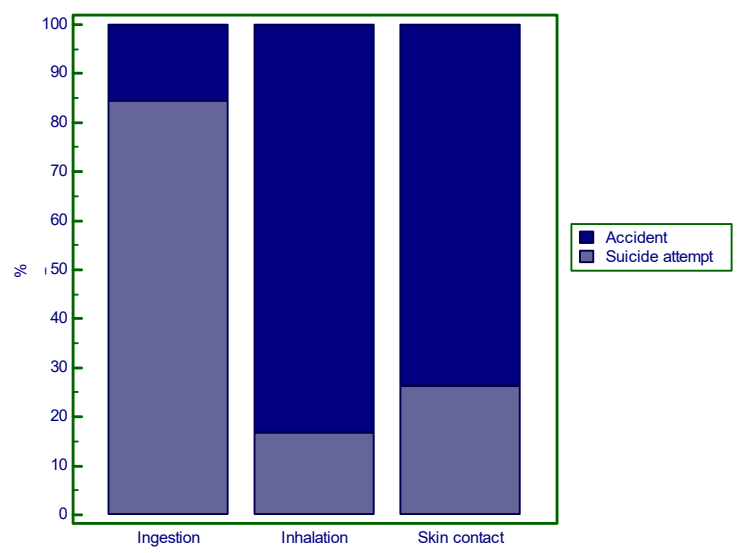

Figure 6 - Cases distribution according to exposure type and intoxication cause

From a paraclinical point of view, measuring the pseudocholinesterase level is a test that reflects the severity of the intoxication. The lower the value of pseudocholinesterase is, the higher the poisoning severity. The pseudocholinesterase values ranged from 540 $\mathrm{U} / \mathrm{L}$ to $10274 \mathrm{U} / \mathrm{L}$, with an average of $4717 \mathrm{U} / \mathrm{L}$ and a median of $4301 \mathrm{U} / \mathrm{L}$. The value of pseudocholinesterase reported to the year of admission did not show any statistically significant differences.

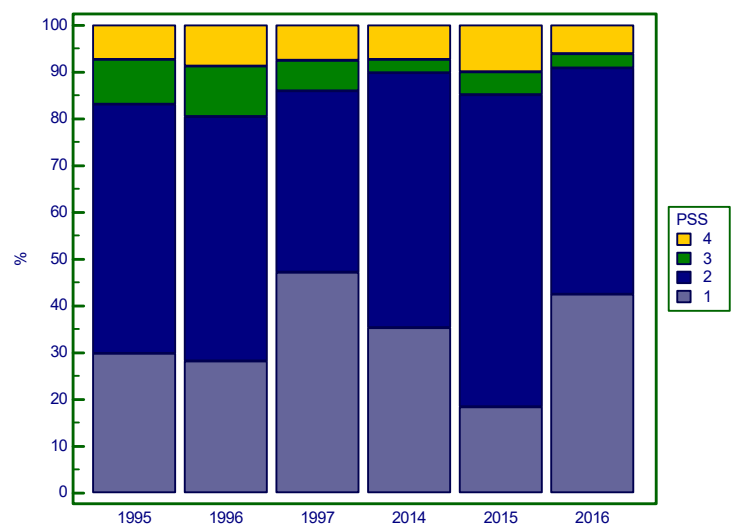

Figure 7 - Cases distribution according to PSS and admission year

The evaluation of the studied group, from the perspective of the PSS, highlights a
PSS of 1 in 153 cases, (33.8\%), a PSS of 2 in 232 cases $(51.2 \%)$, a PSS of 3 in 32 cases $(7.1 \%)$ and a PSS of 4 in 36 cases $(7.9 \%)$. The distribution of patients by years in relation to PSS value does not reveal any statistically significant particularities (Figure 7).

The intoxication severity related to the penetration pathway indicates a low PSS $(\mathrm{PSS}=1)$ in the cases of skin exposure. At the same time, the digestive exposure produced the most serious intoxications (PSS values of 3 and 4). For patients with a PSS of 4 , in addition to digestive exposure, there were identified 1 patient who inhaled OP compounds and 1 patient with skin exposure (Figure 8).

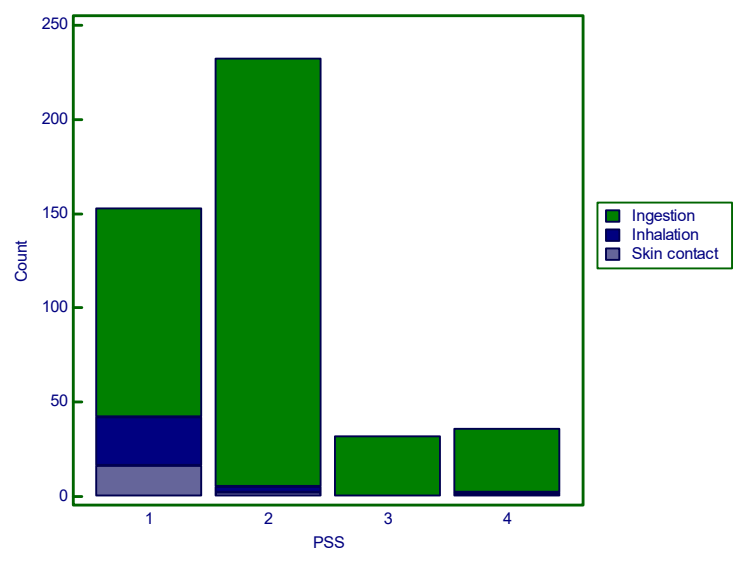

Figure 8 - Cases distribution according to PSS value and exposure type

In the analyzed groups, 36 deaths were identified, with an equal distribution in the two studied periods, of about $8-10 \%$.

\section{DISCUSSION}

The effects of acute poisoning with anticholinesterase agents are manifested by muscarinic and nicotinic signs and symptoms and, with the exception of compounds with extremely low lipid solubility, by signs referring to CNS. Systemic effects occur within minutes after inhalation of vapors or aerosols. Instead, the onset of symptoms is delayed after gastrointestinal and percutaneous absorption. The duration of the effects is largely determined by the properties of the compound: its lipid solubility, the stability of the organophosphorus-acetilcholinesterase bond, and whether or not the phosphorylation enzyme „has aged" [1]. 
Time to death after one acute exposure may vary between five minutes and 24 hours, depending on the dose, the penetration pathway and other factors. The cause of death is primarily respiratory failure, usually accompanied by a secondary cardiovascular component. Muscarinic and nicotinic peripheral actions, as well as central ones, all contribute to breathing stress, which can be manifested by laryngospasm, broncho-constriction, tracheobronchial secretions, increased salivation, voluntary diaphragm and intercostal compromise muscle control, and central respiratory depression. Blood pressure may drop to alarming levels and cardiac arrhythmias may occur. These effects are probably accentuated by hypoxemia. They are often corrected by assisted pulmonary ventilation [2].

Ingestion of OP compounds remains a method of suicide [3]. In our study, this type of contact with OP toxic was the cause of the most severe poisonings. The absorption of OP compounds is high, these substances rapidly bind to plasma proteins. Many compounds are metabolized rapidly, resulting in much more toxic metabolites that aggravate the evolution; paraoxon, malaoxone, diazoxone are active metabolites of parathion, malathion, diazinon [4]. These patients either have associated psychiatric pathology, with repeated previous attempts of suicide, or become patients requiring psychiatric follow-up. Unfortunately, many of these patients refuse to receive qualified help and they end up eventually succeeding in committing suicide. The minimum age at which suicide attempts by ingestion of OP compound were registered was under 16 years of age. This can be closely correlated with the increasingly common forms of depression among adolescents. Also, the maximum age of suicide attempts was extended to over 80 years. In these cases age-related pathologies, neuropsychiatric, oncological, cardiac, as well as poor living conditions, which many elders have in Romania, lack of family affection, lack of support from the state, have an important role.

Confirming the diagnosis of intoxication with OP compounds follows two directions [5], namely:

- gas chromatographic determination of the OP compound or, more frequently, of the metabolites of the compound that caused the intoxication.

- determining the level of cholinesterase. Most commonly determined is plasma cholinesterase. It is possible to determine the cholinesterase from the erythrocyte, less influenced as value by the associated conditions that the patient may have and which are often not known at the time of admission to the department. For the batch studied between 2014 and 2016, plasma cholinesterase was used as a diagnostic factor.

\section{CONCLUSION}

The outcome depends on the level of exposure, the time elapsed between exposure and hospital admission and the rapidity of correct therapeutic intervention. Although there was a 10 -year interval between the two patient lots during which intensive care techniques have significantly progressed, we observed from the statistical data that the death rate did not decrease significantly in the 2014-2016 lot compared to the 1994-1996 lot. We believe that this is partly due to the gravity of these patients and to the time elapsed from the presentation of the patients to the physician and appropriate treatment initiation.

\section{REFERENCES}

[1]D. Z. Hung et al., "The long-term effects of organophosphates poisoning as a risk factor of CVDs: A nationwide population-based cohort study," PLoS One, 2015.

[2] S. S. Peshin, A. Srivastava, N. Halder, and Y. K. Gupta, "Pesticide poisoning trend analysis of 13 years: A retrospective study based on telephone calls at the National Poisons Information Centre, All India Institute of Medical Sciences, New Delhi,” J. Forensic Leg. Med., 2014.

[3] M. Eddleston et al., "Differences between organophosphorus insecticides in human selfpoisoning: A prospective cohort study," Lancet, 2005.

[4] M. B. Colovic, D. Z. Krstic, T. D. LazarevicPasti, A. M. Bondzic, and V. M. Vasic, "Acetylcholinesterase Inhibitors: Pharmacology and Toxicology," Curr. Neuropharmacol., 2013.

[5] S. Chowdhary, R. Bhattacharyya, and D. Banerjee, "Acute organophosphorus poisoning," Clinica Chimica Acta. 2014. 